\title{
CONSIDERATIONS FOR SCIENTISTS GETTING INVOLVED IN OIL SPILL RESEARCH
}

By Kenneth M. Halanych and David G. Westerholm

Oil that reaches the water's surface has already been altered in chemical composition since leaving the well head. Researchers collecting and working with oil should be cognizant of how the environment promotes chemical modifications. Photo courtesy of Georgia DNR




ABSTRACT. From the outset of the Deepwater Horizon (DWH) oil spill, scientists from many different sectors (e.g., government, industry, academia, independent) sprang into action to establish appropriate experimental procedures, collect essential samples, and gather meaningful data. The scale of the spill and the unprecedented use of dispersants challenged scientists familiar with oil spill research, but also drew in many scientists new to hydrocarbon studies. The response to DWH, as with other oil spills, was centered on environmental and human safety concerns as mandated by the US Clean Water Act, the Oil Pollution Act of 1990, and the National Contingency Plan, which defines roles and responsibilities of multiple parties. These roles, however, are usually carried out by government, industry, or government-contracted researchers and, until DWH, have included limited input from academic investigators. In studying the DWH spill, most researchers also had to navigate the logistics and liability issues that can be associated with an oil spill event, including the formal government response processes that can be unfamiliar to academic researchers. In particular, biological researchers had to rapidly educate themselves on the nuances and complexity of the hydrocarbons and dispersants throughout the water column. Nonetheless, biological studies were hampered by the lack of controls or challenges with employing experimental approaches in the field. DWH spill research also highlighted challenges and opportunities that arose due to the interactions of researchers from the academic, government, and industry sectors. The objective of this article is to provide some perspective and to highlight issues that researchers new to the area should consider when approaching oil spill and dispersant studies.

\section{INTRODUCTION}

Scientific response to and study of an oil spill can be a daunting task. It requires a variety of scientific approaches and inclusion of associated response and remediation efforts as well as assessment of impacts across the complex network of biological systems and different hierarchical levels. In the torrent of biological studies after the Deepwater Horizon (DWH) spill, the importance, relevance, and applicability of a given study proved to be dependent not only upon the quality of the biological aspects of the research but also in many instances on how oil spill chemistry and physics were incorporated into the experimental design and interpretation. At the time of a catastrophic event like the DWH spill, the initial urge of many researchers is to immediately start collecting samples or data to understand impacts to the environment. Although sampling habitats prior to encountering oil is invaluable for pre-spill baselines, preparation for and careful thought about collection schemes and experimental design can significantly improve the prospects of producing meaningful scientific products. some of these aspects are obvious, preparing for and embracing them is different than just recognizing they exist.

\section{CONSIDERATIONS}

Consideration 1: Once the event

occurs, researchers must work

within a command structure, so it

is advisable to build collaborations prior to events.

Conducting research in a dangerous, harmful, or uncontrolled environment is a game changer. DWH was unprecedented in its duration and scope. As such, scientists were trying to collect samples at the same time responders were still trying to control or mitigate the spill. Researchers, especially academic researchers, are accustomed to being independent. However, human safety and mitigation of the spill are the first priorities for the command structure overseeing the incident, not research. During the summer of 2010, there were 47,000 response personnel and approximately 7,000 vessels engaged in spill response (Ramseur and Hagerty, 2013). Dispersant applications, in situ burning, oil spill boom deployments, as well as sampling associated with the response and the Natural Resource Damage Assessment (NRDA) process made site access and logistics for independent research difficult and, in some locations (e.g., near the wellhead), impossible.

During this crucial time, a few researchers had access or approval for collecting in restricted areas, whereas most did not. Moreover, the scale of the spill and the extensive use of dispersants drew in many scientists new to this type of research. Being a researcher with access was not a chance process, and potential researchers need to do their best to be prepared for future opportunities. As expected, researchers who were invited in, and thus had access, offered direct relevant experience with Gulf of Mexico organisms and ecosystems or experience with oil spills, or they were US federal agency researchers charged with environmental stewardship or protection 




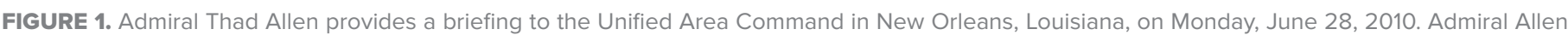

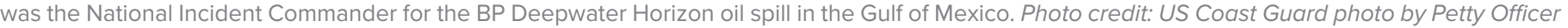
3rd Class Ayla Kelley

(e.g., those affiliated with agencies such as the National Oceanic and Atmospheric Administration or the Environmental Protection Agency). From the outset of the DWH oil spill, scientists from many different sectors (e.g., government, industry, academia, nongovernmental organizations, and independent scientists) were involved in collecting relevant samples and establishing appropriate and meaningful experimental procedures. For an academic researcher, having numerous and strong collaborative ties with scientists in different sectors who know the relevance of proposed research improves chances for access to critical samples or areas. For agencies, promoting openness and transparency can stem some of the mistrust or tension felt by those researchers on the outside looking in.

Understanding the federally mandated response structure provides context for what may or may not be possible. The Oil Pollution Act of 1990 (33 U.S.C. 2701-2761) was enacted in the wake of the Exxon Valdez spill and outlines roles and responsibilities in terms of prevention, response, liability, and compensation for oil spills. This act, in conjunction with federal regulations found in the National Oil and Hazardous Substance Pollution Contingency Plan (NCP; 40 CFR Part 300) establishes response procedures and structures when the federal government responds to an oil spill. Unlike most disaster responses under the US Federal Emergency Management Agency (FEMA) where the federal government often assists local and state responses, under the NCP, the federal On-Scene Coordinator (usually from the Coast Guard or the Environmental Protection Agency) has certain federal authorities. If the On-Scene Coordinator shows that the discharge poses or may present a substantial threat to public health or welfare of the United States, he or she shall direct all federal, state, or private actions to remove the discharge or to mitigate or prevent the threat of such a discharge.

In such a case, the On-Scene Coordinator establishes an Incident Command System, sometimes headed by a Unified Command that employs multiple command posts to oversee containment and response to an incident and guides response at multiple levels (federal, state, local). During the response (Figure 1), a scientific advisor can contribute to the Incident Command System primarily in two ways, as a Scientific Support Coordinator or by working within the "Environmental" Unit. In the case of marine spills, Scientific Support Coordinators will be provided to the On-Scene Coordinator by the National Oceanic and Atmospheric Administration (NOAA). Although they work for NOAA, the Scientific Support Coordinators are to be objective advisors to the On-Scene Coordinator. They have special training and skills and often have established networks of personnel with specific scientific expertise to bring the "best" science to the response. The Environmental Unit, on the other hand, is responsible for executing environmental duties in an Incident Command System structure. It can have numerous personnel assigned with varying expertise. The Scientific Support Coordinator and Environmental teams are necessarily limited in terms of personnel and have specific responsibilities, so most scientists engaged in research on the spill will not be part of these teams. However, if these science advisors are aware of how one's tools or expertise are relevant, chances for access to critical areas or at critical times will be improved. Importantly, Incident Command System personnel work on a timescale much shorter than most researchers. Their first concern is to control the spill with safety in mind. The Oil Pollution Act of 1990 also established the liability framework for the responsible party, including removal costs and damage. In cases like DWH, where the responsible party mobilized and funded the response to remove the oil, industry personnel and resources become part of the Incident Command System structure. This may include industry scientists or contracted scientists with specialties related to the incident. In addition, and often simultaneous to the response, work is done to evaluate the natural resource 
damage and to determine whether the responsible party must compensate the public for damages from the incident and the response (see Consideration 2).

Importantly, one must be qualified to work in such areas when the time comes. In hazardous situations, this often requires certifications and knowledge of legal and safety protocols that are best obtained prior to the event. Once the event happens, there can be confusion about where and how to be trained for working in hazardous situations, and even a backlog of requests for training. An excellent primer for academic researchers has been developed by NOAA's Sea Grant (Sea Grant Programs of the Gulf of Mexico, 2020).

\section{Consideration 2: Source of}

\section{research support.}

Duringaresponse, limitedsampling (water, air, sediment) may be conducted by the Unified Command/Incident Command if it is directly related to the response. If alternative countermeasures are going to be utilized, Special Monitoring of Applied Response Technologies (SMART) protocols and monitoring are employed. For example, during in situ burning or dispersant operation, rapid, real-time sampling and reporting provides the Unified
Command/Incident Command with data for decision-making. Although SMART is not a regulatory requirement, scientists assist with reporting and evaluating the data collected.

In concurrence with DWH response efforts, a number of researchers were also mobilized to conduct a Natural Resource Damage Assessment. NRDA is a legal process under which designated natural resource trustees (from federal, state, and tribal governments with jurisdiction) determine the appropriate type and amount of restoration needed to offset impacts and lost use to fisheries, wildlife, habitats, and human uses from oil spills, hazardous waste sites, and vessel groundings. NRDA occurs in phases that include (1) assessment of the injury (which may include a preliminary assessment), (2) planning the restoration, (3) holding polluters accountable (via settlement or court action), and (4) restoring the environment to the extent practicable. This work is usually done by government scientists and specialized contracting firms or institutions that conduct NRDA studies. However, for the DWH spill, the government turned to academic researchers more than usual because of the scale of the spill and because government scientists, consultants, and industry researchers had limited knowledge of deep-sea environments. In such a situation, the academic researchers are formally contracted and typically must sign a non-disclosure agreement. In some cases, this fostered tension between academic researchers. Such non-disclosure agreements should be vetted carefully by the parties involved (including university lawyers), as publication of results may be prohibited for some length of time, impacting the research outputs of principal investigators and, importantly, early career scientists in their laboratories. Fortunately, in the case of DWH, the government realized that the peer-review process bolsters the integrity of a scientific study, making findings more likely to hold up to scrutiny in a court of law.

Unlike the response effort, which may be of relatively short duration, NRDA can often take years to complete. In the case of DWH, the NRDA settlement was approved nearly six years after the historic event, and restoration efforts will continue until 2032. Researchers may be involved throughout this process. At the same time, the responsible party will also independently evaluate damages, a process that may be done with designated

\section{BOX 1. The Benefits of Industry-Academia Interaction in Oil Spill Research}

During the DWH spill, interactions between researchers in different sectors were typically positive and productive, but several interactions, especially between industry and academia, were initially challenging. Industry scientists often have knowledge of considerable research that is not available to the general community. This situation can cause tension, as in some cases industry researchers can be dismissive because they think the work was already completed, and academicians are skeptical about the quality of nonpeer reviewed, or privately reviewed, work. As was clear in the case of research on dispersants, considerable insights and knowledge were gained from a fresh perspective that academic researchers brought to the table, and academic researchers benefited from industry's knowledge. Moreover, all researchers who participate in spill response must be open and realistic about their biases and motives versus the biases and motives of others. In most cases for DWH research, once researchers interacted in a neutral setting, a collaborative mindset ensued. For example, larger research consortia that comprised much of the Gulf of Mexico Research Initiative's (GoMRl's) effort included industry representatives who served on their advisory boards. The interaction between sectors can help industry scientists see beyond there company-driven perspective and can help academic scientists from repeating research efforts already carried out by companies. Transparency and communications are fundamental to facilitating such interactions. Funders of oil spill response and research can serve a critical role in developing these interactive relationships, as was done in the GoMRI research model. 
national trustees in a cooperative manner (i.e., joint research and sharing of data, with each party conducting independent evaluations) or, if not, the responsible party may choose scientists or contracted researchers. Because this is a legal process, chain-of-custody protocols and limitations on researchers will often apply until the process is complete.

To help mitigate conflict-of-interest issues, most NRDA science is contracted to companies that specifically support the government or those that specifically support the industry responsible parties, and not to independent sources. This model also includes sample analysis, where specialized and certified laboratories can meet the legal requirements. A nuance is that if data are publicly available, such as those published by a researcher, the government and/or industry may choose to use that information as it relates to potential injuries to natural resources. A poignant example is the discussion of flow rates from the wellhead during the spill, which sparked disagreements
Although the NRDA covers damages incurred to public resources, it does not get involved with commercial claims. The responsible party usually sets up a claim process for third parties. In large-scale or unusual events, there may be an opportunity for specific research associated with those commercial claims, either to support those claims against the responsible party or to refute the claims on behalf of the responsible party.

DWH was unique in that there were significant research funds available spread out over many years. Examples include US National Science Foundation Rapid Response Research (RAPID) grants and Gulf of Mexico Research Initiative (GoMRI) funding (a 10-year program that was not required by law or settlement), as well as Resources and Ecosystems Sustainability, Tourist Opportunities, and Revived Economies of the Gulf Coast States (RESTORE) Act and National Academies funding that continue today. Future spill events may provide similar opportunities for academic research
Lastly, one overarching issue concerning support for DWH research was that those who had baseline data or ongoing studies were targeted by research agencies and the NDRA process. As mentioned below, having before and after comparisons is essential for understanding environmental impact.

\section{Consideration 3: Liability.}

Academic researchers understand that there is liability with field research or sample collection, but the focus is usually on an individual's well-being or equipment used in the field. In the case of an oil spill, there are several other aspects to liability as well. Under the Oil Pollution Act of 1990, the company that caused the spill is the financially responsible party for impacts caused by the spill including any impacts that may have been caused by response to, or assessment of, the spill.

In other words, if a researcher is somehow injured in response to the spill, exposed to toxicants, or causes further harm to the environment, including

\section{Focusing on the oiled communities and environment, rather than other objectives or priorities relevant to specific research sectors, is the best way for all to benefit from research efforts.}

among NRDA-contracted researchers, federal agencies, and industry representatives regarding damages wrought by the spill. In extremely rare cases, for example if the case were to go to trial, researchers may be requested to provide depositions or asked to be witnesses if their material is particularly germane. Such material may include field samples, cruise logs, laboratory notebooks, instrument data files, and email correspondence. funding. Congress can also play a role by providing federal funding and mandating research from certain agencies, which usually occurs post event. Of course, there is unassociated research that may link to an event, such as fisheries stock research, seafood safety research, and long-term environmental studies that now have oil introduced to their study area and that may have long-term implications for resource management. causing delays in the response, the responsible party for the spill may incur additional liability. Likewise, research results may either directly or indirectly inflate assessments of damages caused by the spill. For example, findings that are publicly vetted and cause further damage to local economies (e.g., by reducing beach tourism or reductions in recreational fishing) may incur additional third-party claims or additional NRDA 
injuries. In either of these cases, the responsible party may seek to attribute such damages to researchers or their institutions and seek third-party compensation. In this vein, how findings and results are reported should be considered. Peer-review publications have been vetted by qualified individuals and the language focuses on accurate and scientifically supported statements. In contrast, a university press office or a public media outlet may try to reduce statements to more simple or catchy language that may be interpreted in unintended ways. Understanding these potential liabilities can be critical for making sure the proper steps are taken to ensure actions or reported findings are done in a manner that reduces the exposure of the researchers and their institutions.

\section{Consideration 4: Design}

research to fit into an appropriate comparative framework.

The importance of controls for comparison is well known to researchers, but during the DWH spill studies were hampered by the limited availability of baseline data or appropriate control samples to ensure proper experimental design. Biological systems, from organism to ecosystem, are subject to multiple stressors (e.g., Carmichael et al., 2012; Weinnig et al., 2020). This especially is true in the Gulf of Mexico, a system known for variability (e.g., Morey et al., 2005; Kolker et al., 2011; Xue et al., 2013; MullerKarger et al., 2015). Without sufficient resources to appropriate governmental agencies and other stakeholders, obtaining a baseline understanding of the contaminant burden of the ecosystem and the degree of underlying variability will remain elusive.

Baseline data prior to DWH was remarkably scarce with respect to important issues such as background levels of polycyclic aromatic hydrocarbons (PAHs), organism health of important species, or even variations in community structure and biodiversity. In some cases, data that were available were not easily discoverable. When baseline data were available, the collection method or frequency may have been different from what was needed after the event, compromising the utility of such data. Although several studies focusing on DWH used comparative baseline data, interpretation of impacts may have been hindered due to the difficulty of elucidating influences of multiple simultaneous stressors (Chagaris et al., 2020). The more meaningful studies that used baseline comparisons were largely either continuations of previous studies or they took advantage of previously collected samples (e.g., Pulster et al., 2020).

Without baseline data, many researchers employed a modified before-aftercontrol-impact (BACI) experimental design (Conner et al., 2016; Smokorowski and Randall, 2017). In the case of DWH, this mainly focused on the control versus the impact of the experimental design because "before" data were limited or lacking. One challenge of this particular spill was that the scale was so large that finding appropriate controls (i.e., sites that were not impacted) in close proximity and with similar parameters was difficult.

\section{Consideration 5: Experimental \\ design should account for \\ the chemical complexity, and \\ environmental state, of oils \\ and dispersants.}

Oil is a complex mixture of hydrocarbons and the result of multifaceted geological processes. As such, the composition of oil varies significantly depending on its source. The sizes of oil molecules emanating from a wellhead may range from small ones such as methane $\left(\mathrm{CH}_{4}\right)$ and ethane $\left(\mathrm{C}_{2} \mathrm{H}_{6}\right)$ up to larger asphaltenes, whose molecular masses can be as much as 1,500 u (Rullkötter and Farrington, 2021, in this issue). Oil can be composed of hydrocarbon chains or molecules that form rings and can be saturated or unsaturated. Because of this complexity, the exact molecular composition of a particular oil sample is very difficult to know. Oil fingerprinting depends on identifica- tion using a combination of key hydrocarbon compounds that are uniquely tied to the oil's source. Importantly, some molecules in oil are labile and others recalcitrant, meaning that the composition of oil changes over time. For example, in the case of $\mathrm{DWH}$, much of the methane found in the oil mixture emanating from the wellhead on the ocean floor never made it to the ocean surface, as it was absorbed into the water column. Likewise, once the oil reached the photic zone, photo-oxidation of hydrocarbon molecules began changing the nature and composition of the oil. As a result, oil that has undergone modification in the environment, that is, "weathered oil," can be compositionally very different than oil released at the wellhead.

In contrast, the chemical formulations of dispersants are known and can be reproduced. However, because they are proprietary, the researcher conducting experiments may not have knowledge of the precise formulas used during the spill response. Although we suspect that molecular components of dispersants also change under environmental conditions, we have much less understanding of the longevity of such molecules in the environment and how they degrade or are catalyzed into subsequent entities. Tracking them in the environment can be more difficult than tracking hydrocarbons (Quigg et al., 2021, in this issue).

Biological researchers need to do a better job of considering the chemical and physical properties of molecular components of oil and dispersants to gain a more accurate and thorough understanding of hydrocarbon and dispersant impacts on biological systems. For example, differences in hydrocarbon composition can impact carbon assimilation rates by microbes that feed on the oil deep in the water column versus on the surface (Weiman et al., 2021, in this issue; see photo on this article's opening page). Such differences in carbon utilization are important, as carbon from DWH oil was directly taken up into food webs (Graham et al., 2010; Chanton et al., 2012; 


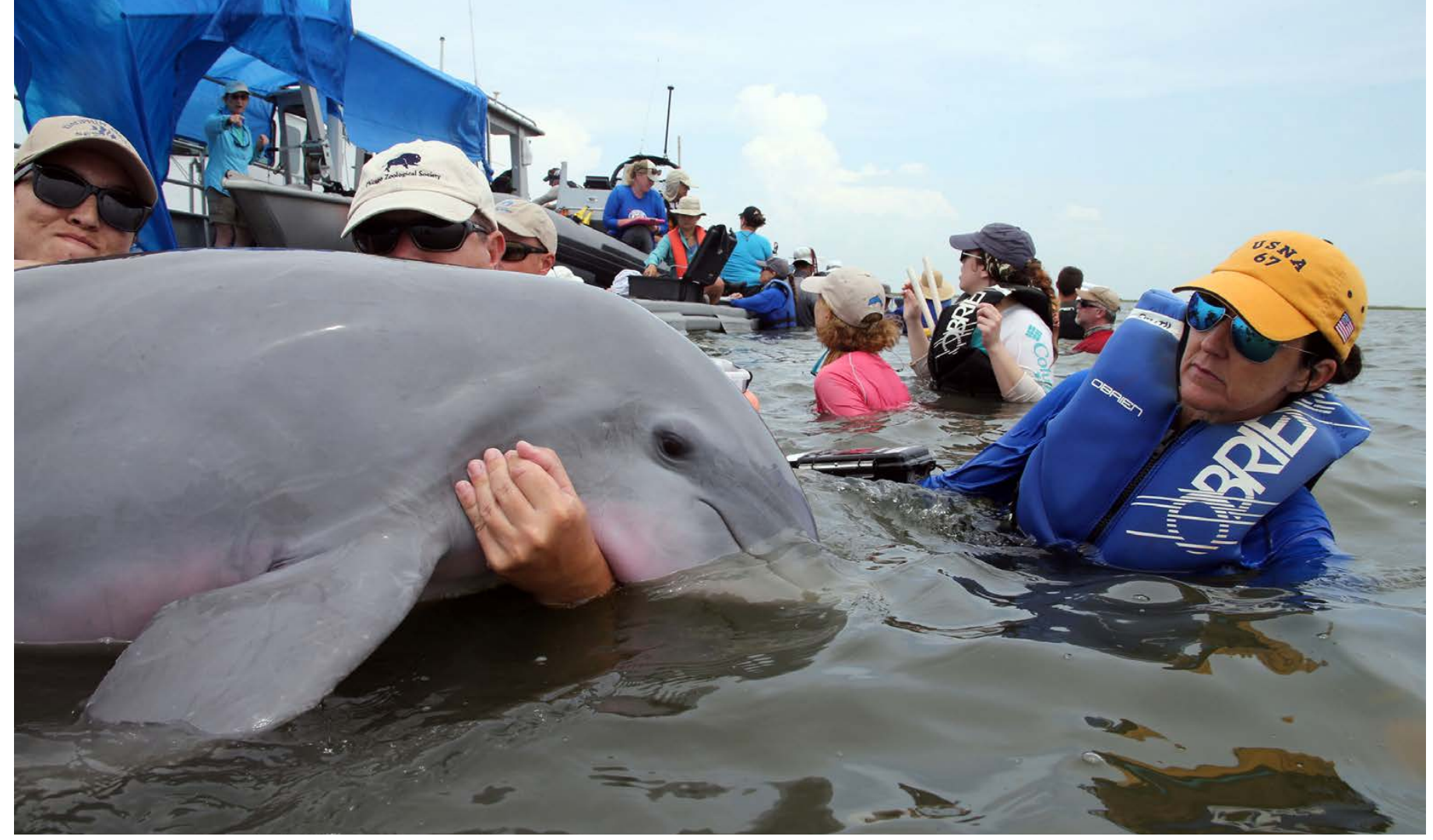

FIGURE 2. Scientists monitored mobile animals such as dolphins, which experienced wide variation in toxicant exposures. Photo credit: Consortium for Advanced Research on Marine Mammal Health Assessment

Quintana-Rizzo et al., 2015). Using similar logic, DWH sourced oil may have limited applicability to some questions addressed in oil pollution studies due to compositional differences in oil chemistry.

Immediately following the DWH spill, many researchers scrambled for access to Macondo well oil, or similar surrogates, to use in experiments. Fresh or "pristine" oil was used in experiments for shallow and nearshore water and for terrestrial organisms, even though these animals were only exposed to weathered oil that was reduced in several of the smaller volatile hydrocarbons (Rullkötter and Farrington; 2021; Farrington et al., 2021, both in this issue). Critically, different compounds in oil and dispersants have differential toxicity and can produce various secondary compounds. Healthy skepticism is warranted when assessing how well a particular study has replicated the hydrocarbon or dispersant environment of the biological system under study.

Thus, when conducting research, investigators should consider (1) the composition of oil and/or dispersants that the organismal system was exposed to, given modification by "weathering," and (2) which of the hundreds to thousands of chemical compounds found in oil and dispersants are likely responsible for the observed organismal response. Because accurate assessment of oil and dispersant composition is beyond the scope of most biologists, a researcher should consider collaborating with an appropriate chemist, preferably one with mass spectrometry experience. Because many previous studies have treated oil (and/or dispersant) as a singular entity, many of the molecular mechanisms that actually cause harm or insult to biological systems remain unknown.

\section{Consideration 6: The scale and magnitude of parameters and results to be measured should be carefully considered.}

Major catastrophic events have considerable lethal impacts that are often quicker and easier to measure than sublethal and long-term effects. In the case of DWH, oil and dispersants were distributed over hundreds of square kilometers, and concentrations of specific hydrocarbon molecules or dispersant components varied spatially and temporally on scales that spanned several orders of magnitude. Although much of this was driven by the movement of oil, organisms also moved in and out of impacted areas. For example, pelagic organisms characterized by diel vertical migrations (Sutton et al., 2020) and highly mobile mammals (Figure 2) likely moved through different concentrations of the oil.

In designing experiments and reporting results, researchers should be as explicit as possible in replicating environmental conditions of interest. Also, for results to be more robust and meaningful, researchers should consider experimental designs that encompass a broad range of dosages for the pollutant in question. For example, if a nearshore fish species is being examined in a mesocosm, is the experimental design trying to replicate pollutant conditions observed at the edge of a large slick, or conditions where the oil has been broken into small droplets 
suspended midwater, or something else? In these cases, the organism may have been exposed to varied pollutant concentrations, and the exposures may have been more episodic than constant in nature. If such differences in exposure are not considered during interpretation of the data, extrapolation to impacts of the spill on the health of individuals or populations may be flawed.

The remainder of this article focuses on some of the lessons learned and approaches that have continued to be refined over the 10-year course of DWH research.

\section{RESEARCH LOGISTICS}

Research related to an oil spill has some unique considerations. Although government, industry, and academic investigators may have similar reasons for doing research (i.e., to understand how the spill impacted the environment), their uses of data can be very different (e.g., peerreviewed publications, industry reports, governmental assessments, or court proceedings). Many scientists may contribute to NDRA research to inform damage assessments that will be critical to settlement negotiations or court cases. Some researchers became involved in the DWH spill due to a sense of civic duty or because it was a logical extension of ongoing work, and for some, it presented a funding opportunity. Regardless of the motivation for conducting the work, how the research is to be used can impact logistical considerations. For example, research under the NRDA umbrella requires a strict accounting of the chain of custody for samples and more rigorous standards of laboratory documentation than may be customary for academic scientists. Some of the logistical considerations are outlined below.

\section{Logistical Consideration 1: Chain of} custody and documentation.

If the research is part of an NRDA process, then chain-of-custody documentation for samples is usually required.
However, even if the research is not part of the damage assessment, using a chainof-custody approach may be advisable. Environmental disasters caused by human activity attract multiple stakeholders (e.g., industry, governments, environmental groups, local residents), some of whom may attack a given research finding based on their interests rather than the quality of science. Additionally, even if researchers are not initially involved in damage assessment, they may possess unique samples or results of interest to formal proceedings at a later date. Alternatively, the responsible parties may seek compensation if they feel research inappropriately contributed to their liability exposure. Thus, the work may need to be defensible in a legal, in addition to a scientific, framework. As such, a researcher wants the work to be as defensible as possible, including verifying explicit details of procedures used in the lab and who had access to samples at all times. This may require signed research logbooks or having samples under lock and key in one's own lab.

\section{Logistical Consideration 2:}

\section{Plastic is a hydrocarbon.}

The use of plastics is ubiquitous in biological research settings, largely because they are durable and convenient, but components used in making plastic containers and Eppendorf tubes, for example, may be similar to the hydrocarbons found in oil. If measurements are to be made of hydrocarbon concentrations in tissue, items used in the transport and storage of samples, or housing of live organisms, should be considered. Plastics may not be appropriate, and containers made of glass or foil may be preferred. This consideration is particularly relevant for eliminating contamination in experimental design where measurements for sublethal exposure to oil products may be in the parts per billion, or in working with dispersants where contamination is possible from similar compounds that can be found in a working laboratory setting.
Logistical Consideration 3:

Replicating environmental

parameters will determine the

experimental setting and scale.

Because both oil and biological systems are exceedingly complex, and dispersants are somewhat of an unknown quantity, the number of parameters to manage when conducting oil-spill research is potentially unwieldy. Much of the success of DWH research was facilitated by using a tiered approach to handle and replicate biological complexity. A combination of lab, mesocosm, and/or field experiments was used to relate parameters that could be clearly defined in a controlled setting to more realistic environmental settings (Coull and Chandler, 1992). In the case of oil spill research, experiments in the field are not only difficult because of the number of parameters that are free to vary, but the introduction of pollutants that exceed regulatory thresholds (e.g., oil sheen) is often unethical and usually prohibited by law.

In the laboratory, experiments can focus in on impacts of oil and dispersants by holding all other parameters constant. Although this works well for specific questions regarding individual organisms, this approach usually is too restrictive to deal with how oil impacts ecological connections between organisms. Thus, to provide a more complex but controlled environment, mesocosms have been very helpful as they allow for a greater, but still manageable, number of parameters to vary or be subject to manipulation. The questions driving the research, along with careful consideration of the environmental conditions to be examined, will help determine how lab, mesocosm, and field experiments should be employed.

\section{Logistical Consideration 4:}

Toxicity and waste management.

Just like the handling of any other toxic substance used in an experimental setting, disposing of hazardous waste must be considered. Many DWH researchers were innovative in their experimental designs, which in some cases required 
new solutions to hazardous waste management. Regardless of whether one is using a $250 \mathrm{~mL}$ flask in the lab or a $20,000 \mathrm{~L}$ mesocosm, water treated with pollutants must be disposed of properly and equipment used must be properly cleaned. Although this may be a minor issue for small aquaria, cleaning and hazardous waste protocols can be expensive both in terms of time and money for larger facilities. Before hastily starting an experiment because samples are available or there is a need to act quickly, researchers should make sure they have adequately planned for disposal of waste and wastewater and for how equipment will be cleaned or detoxified. Because biosafety, chemical safety, and other safety offices have their own schedules, planning and contingencies are best done before crisis hits, if at all possible.

\section{Logistical Consideration 5:}

Delivery and dosing of organisms with oil and dispersants is not a trivial procedure.

As indicated above, over the course of the 10-year GoMRI program, researchers have learned a considerable amount about the best ways to study the impacts of oil spills. Because oil and dispersants are complex chemical mixtures, and because oil and water don't mix (to use an age-old phrase), delivering oil and dispersants to organisms in the desired quantities can be a challenge. For example, if oil is added to an aquarium containing a crab, the oil will likely float. In that case, determining which components and what concentrations of the oil may leach into the water and reach the crab at the bottom of the tank requires additional measurements and understanding of chemis- try. Similarly, the water-oil interface in a plexiglass versus a glass tank can be different. Such factors can alter organismal interactions with toxicants.

Methods for delivering oil and dispersants to study organisms have been refined over many years. Water accommodated fractions (WAFs) for hydrocarbons and chemically enhanced water accommodated fractions of oil (CEWAFs) have been used for the combination of hydrocarbons and the Corexit dispersant that was used during DWH. The techniques used for dosing oil and dispersants, including which chemicals in those mixtures are released, need careful consideration and can present myriad challenges (e.g., Wade et al., 2017; Bera et al., 2018; Hodson et al., 2019; Colvin et al., 2020; NASEM, 2020).

\section{CONSIDERATIONS FOR INTERPRETATION}

Consideration for Interpretation 1: Disentangle effects due to different molecules.

A significant challenge during the DWH research effort was separating impacts due to hydrocarbon exposure versus dispersant exposure. One issue was that good estimates of exposure concentrations of dispersants in animals were largely unknown (NASEM, 2020; Quigg et al., 2021, in this issue). However, in the laboratory, many researchers quickly gravitated to experimental setups that exposed different cohorts to either oil, dispersant, or a combination of dispersant and oil (with appropriate controls; Figure 3). Due to the complexity of these mixtures and ongoing physical-chemical processes during the experiment, determining which chemicals within oil or dispersants are likely to be mechanistically responsible for causing an observed change in organismal state can be very difficult.

During analyses and interpretation, researchers should take care to clearly articulate the resolution at which interpretations can be drawn. For example, based on how a given study was conducted, can we say that the impact was due to hydrocarbons in general, PAHs, or a single specific chemical?

\section{Consideration for Interpretation 2: Recovery is not just a function of time but of processes.}

As mentioned, oil and dispersants are a complex cocktail of molecules, many of which can be degraded or metabolized, thus changing the "cocktail" over time,

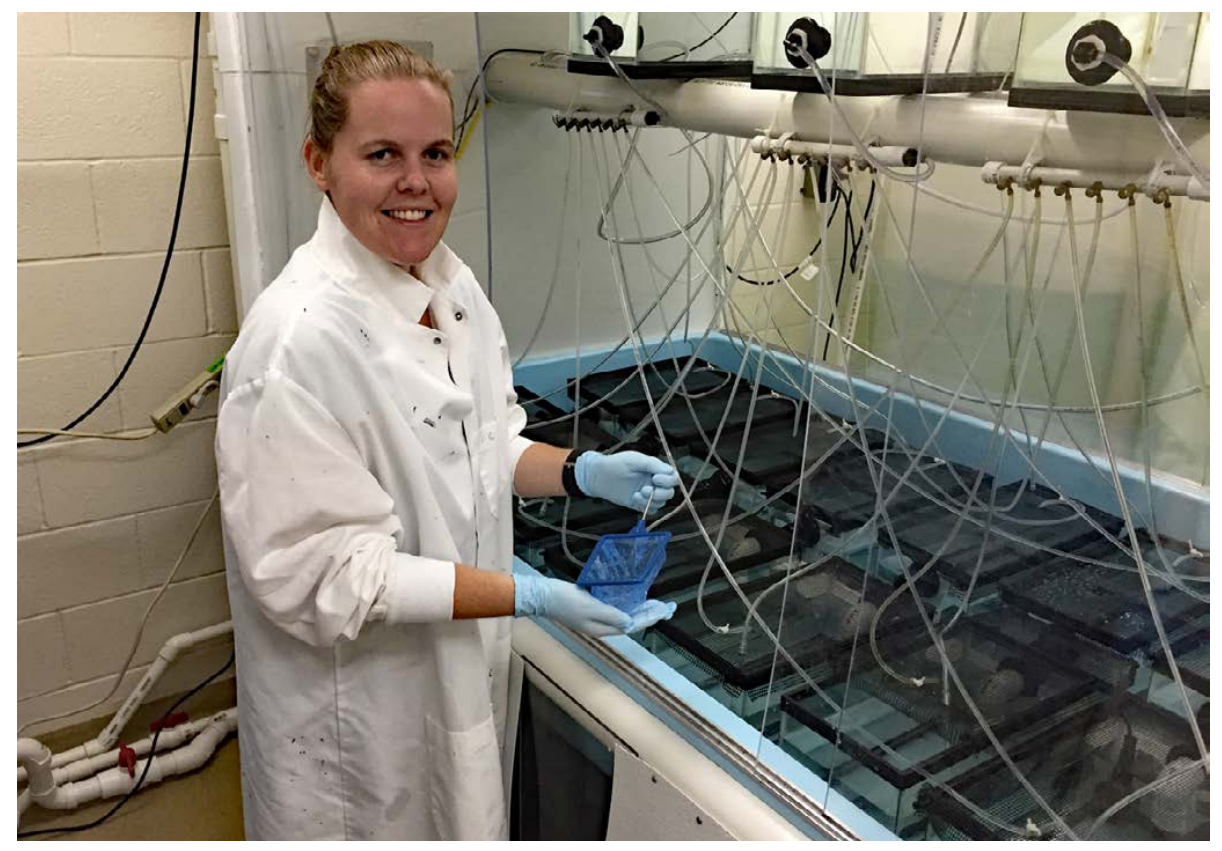

FIGURE 3. Working with oil in a controlled set ting offers many challenges in terms of replicating organismal exposure, chemical modification, and disposal of hazardous substances. Here, a researcher works at an aquarium designed to replicate marine animal oil exposure and multiple environmental stressors. Photo credit: Milton Levin 
even in some laboratory and mesocosm experiments (Farrington et al., 2021, in this issue). Therefore, when looking for responses of biological systems over time, focusing on a single indicator or marker may not be sufficient. Importantly, toxicants can have sublethal impacts that take time to manifest. Chemical components of hydrocarbons and dispersants can be metabolically active and produce secondary compounds. The initial indicator molecule measured in the environment may have been transformed into compounds that are more or less detrimental.

For sublethal or long-lasting impacts, rates of processes that control cellular metabolism may be much better indicators of recovery than time since event. For example, $\mathrm{PAH}$ metabolites measured in the tilefish Lopholatilus chamaeleonticeps increased over time following the DWH event (Snyder et al., 2019; Figure 4). These PAH metabolites were positively correlated with decline in fish health and appear to have been taxing the energy budgets of individuals, particularly of adult females, and ultimately impacting fitness. Additionally, the reproductive lifespan for many deep-sea and larger nearshore organisms is typically much longer than the duration of the NRDA process or other research programs, including the GoMRI effort that lasted 10 years. The example of metabolite load in tilefish resulting from the initial toxicant is illustrative, as that study included five years of sampling. Thus, referring to a rate of degradation or the accumulation of a metabolite, rather than measuring the amount of a particular toxicant, may be more useful for tracking recovery.

\section{CONSIDERATIONS FOR FUTURE ENGAGEMENT}

Academic researchers who want to be involved in spill research may find funding more difficult after time has elapsed from a major event. However, engaging with the response and planning community may still provide insight and opportunity. Federal agencies that are engaged in spill response are part of the

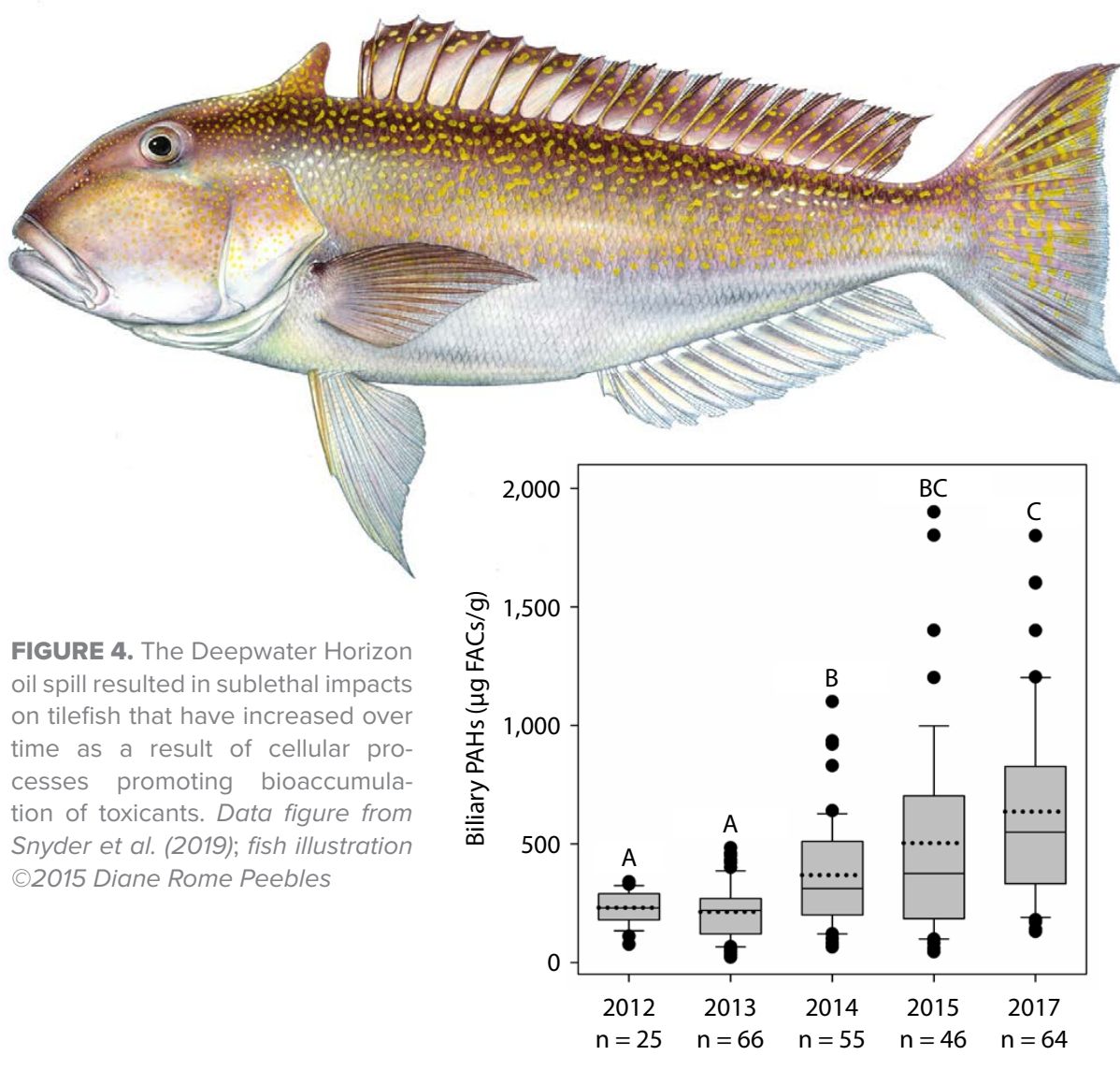

Interagency Coordination Committee for Oil Pollution Research (ICCOPR), which reports to Congress on funded research projects as well as unmet needs. Additionally, for the marine environment, the Coast Guard has Area Committees comprised of federal, state, and local officials whose responsibility is to prepare an Area Contingency Plan for a response to a discharge of oil or hazardous materials. For many academic researchers, the most appropriate intersection with oil spill response may be through open meetings held by Area Committees. In addition to governmental agencies, numerous individuals from oil spill response organizations, industry, academia, and environmental groups participate in these committees' planning processes. Understanding what is planned for a major spill can bring focus to research areas and familiarity with those who will be leading the response. This is a process where academic researchers can share insights with colleagues from other sectors and collectively bring the latest knowledge of a particular research area to the framework of response, NRDA, and restoration planning as well as identify ongoing or new needs for advancement of knowledge. Connecting to these groups can be facilitated by NOAA and the US Fish and Wildlife Service as well as the Coast Guard and NOAA Sea Grant.

Importantly, current laws and regulations mandated by the Clean Water Act, the Oil Pollution Act of 1990, and the National Contingency Plan undervalue the importance of the academic sector to the science and remediation of largescale oil spills. Without modification or changes based on the DWH experience, the next major event will likely see similar confusion and inefficient use of resources at the outset. Government agencies and universities must engage with legislatures to explain the insight and potential that academicians bring to the table. At the same time, revisiting and revising laws to promote positive and progressive interactions among industry, academic, and government sectors during such an 
event would provide a substantial benefit for response, mitigation, and restoration of future disasters.

The need for an efficient communication infrastructure by the Incident Command System and responding agencies should be a critical consideration for future events. Well-established lines of communication that promote transparency and have sufficient capacity will only benefit all involved. DWH was so massive compared to previous incidents that established channels of communication could not handle the large volume of information and communications needed. These limitations sowed confusion and, in some cases, distrust. The lack of sufficient bandwidth impacted researchers, but more importantly, increased and promoted anxiety for some sectors of the public. Mandates for expanding the team of science advisors, as well as specialists to engage the public, should be explored.

One last consideration: expect the unexpected, as there will be unforeseen processes, and every spill is different. The great thing about research is that there are always new discoveries and surprises. Be open to and prepared for unexpected findings, including some that do not fit preconceived models or ideas. Early in the DWH spill there were, of course, comparisons to the other major spill in US waters, the Exxon Valdez. However, that was a surface spill of heavy crude oil, in contrast to the DWH spill, which consisted of a sweet light crude that occurred at great depth below much warmer surface waters. Comparatively, the oil and associated chemicals from DWH were metabolized relatively quickly by microbes (Weiman et al., 2021, in this issue). The 1979 Ixtoc 1 spill in the Bay of Campeche in Mexican waters, also a prolonged major spill in the Gulf of Mexico, was more similar in many ways to DWH. However, several factors relating to funding, accessibility in Mexican waters, and decisions about how research finding would be disseminated limited the impact on scientific knowledge that resulted from Ixtoc 1. All three of these major North American spills were important for advancing understanding of the environmental impacts of spills. Oil spills differ in a number of ways, and thus there will always be some degree of unpredictability.

\section{SUMMARY}

Although the target audience of this article is largely those new to oil spill research, there are several points that apply to a broad cross section of investigators. A major oil spill causes a complex cacophony of interactions, not only in the environment, but among response teams and investigators. Being prepared to work efficiently in such an environment is no accident, and preparatory steps can be taken to improve the possibility of conducting meaningful research in such a situation. Successful participation in that cacophony requires being able to work with, and learn from, individuals in other research sectors. Investigators in government, industry, or academic positions all have their own strengths and perspectives. Focusing on the oiled communities and environment, rather than other objectives or priorities relevant to specific research sectors, is the best way for all to benefit from research efforts. Lastly, oils spill research is exceedingly interdisciplinary. To address even a simple question in a specific and thorough manner, researchers need help from other disciplines, especially chemistry. All of the issues discussed here developed and matured over the course of DWH research, and the hope is that the knowledge gained during 10 years of Gulf of Mexico Research Initiative efforts can jumpstart research on the next spill. @

\section{REFERENCES}

Bera, G., T. Parkerton, A. Redman, N.R. Turner, D.A. Renegar, J.L. Sericano, and A.H. Knap. 2018 Passive dosing yields dissolved aqueous exposures of crude oil comparable to the CROSERF (Chemical Response to Oil Spill: Ecological Effects Research Forum) water accommodated fraction method. Environmental Toxicology and Chemistry 37:2,810-2,819, https://doi.org/10.1002/ etc. 4263 .
Carmichael, R.H., W.M. Graham, A. Aven, G. Worthy, and S. Howden. 2012. Were multiple stressors a 'perfect storm' for northern Gulf of Mexico bottlenose dolphins (Tursiops truncatus) in 2011?

PLoS ONE 7:e41155, https://doi.org/10.1371/journal. pone.0041155.

Chanton, J.P., J. Cherrier, R.M. Wilson, J. SarkodeeAdoo, S. Bosman, A. Mickle, and W.M. Graham. 2012. Radiocarbon evidence that carbon from the Deepwater Horizon spill entered the planktonic food web of the Gulf of Mexico. Environmental

Research Letters 7(4):045303, https://doi.org/ 10.1088/1748-9326/7/4/045303.

Chagaris, D.D., W.F. Patterson III, and M.S. Allen. 2020. Relative effects of multiple stressors on reef food webs in the Northern Gulf of Mexico revealed via ecosystem modeling. Frontiers in Marine Science 7:513, https://doi.org/10.3389/ fmars.2020.00513.

Colvin, K.A., C. Lewis, and T.S. Galloway. 2020. Current issues confounding the rapid toxicological assessment of oil spills. Chemosphere 245:125585 https://doi.org/10.1016/j.chemosphere.2019.125585.

Conner, M.M., W.C. Saunders, N. Bouwes, and C. Jordan. 2016. Evaluating impacts using a BACl design, ratios, and a Bayesian approach with a focus on restoration. Environmental Monitoring and Assessment 188:555, https://doi.org/10.1007/ s10661-016-5526-6.

Coull, B.C., and G.T. Chandler. 1992. Pollution and meiofauna: Field, laboratory, and mesocosm studies. Oceanography and Marine Biology: An Annual Review 30:191-271.

Farrington, J.W., E.B. Overton, and U. Passow. 2021. Biogeochemical processes affecting the fate of discharged Deepwater Horizon gas and oil: New insights and remaining gaps in our understanding. Oceanography 34(1):76-97, https://doi.org/10.5670/ oceanog.2021.118.

Graham, W.M., R.H. Condon, R.H. Carmichael, I. D'Ambra, H.K. Patterson, L.J. Linn, and F.J. Hernandez Jr. 2010. Oil carbon entered the coastal planktonic food web during the Deepwater Horizon oil spill. Environmental Research Letters 5:045301, https://doi.org/ 10.1088/1748-9326/5/4/045301.

Hodson, P.V., J. Adams, and R.S. Brown. 2019. Oil toxicity test methods must be improved. Environmental Toxicology and Chemistry 38:302-311, https://doi.org/10.1002/ etc. 4303.

Kolker, A.S., M.A. Allison, and S. Hameed. 2011. An evaluation of subsidence rates and sea-level variability in the northern Gulf of Mexico. Geophysical Research Letters 38:(21), https://doi.org/10.1029/ 2011 GL049458.

Morey, S.L., J. Zavala-Hidalgo, and J.J. O'Brien. 2005. The seasonal variability of continental shelf circulation in the northern and western Gulf of Mexico from a high-resolution numerical model. Pp. 203-218 in Circulation in the Gulf of Mexico: Observations and Models. Geophysical Monograph Series vol. 161, W. Sturges and A. Lugo-Frernandez, eds, American Geophysical Union, Washington, DC, https://doi.org/10.1029/161GM16.

Muller-Karger, F.E., J.P. Smith, S. Werner, R. Chen, M. Roffer, Y. Liu, B. Muhling, D. Lindo-Atichati, J. Lamkin, S. Cerdeira-Estrada, and D.B. Enfield. 2015. Natural variability of surface oceanographic conditions in the offshore Gulf of Mexico. Progress in Oceanography 134:54-76, https://doi.org/10.1016/j.pocean.2014.12.007.

NASEM (National Academies of Sciences, Engineering, and Medicine). 2020. The Use of Dispersants in Marine Oil Spill Response. The National Academies Press, Washington, DC, 340 pp., https://doi.org/10.17226/25161. 
Pulster, E.L., A. Gracia, S.M. Snyder, K. Deak, S. Fogelson, and S.A. Murawski. 2020. Chronic sub-lethal effects observed in wild-caught fishes following two major oil spills in the Gulf of Mexico: Deepwater Horizon and Ixtoc 1. Pp. 388-413 in Deep Oil Spills: Facts, Fate, and Effects. S.A. Murawski, C.H. Ainsworth, S. Gilbert, D.J. Hollander, C.B. Paris, M. Schüter, and D.L. Wetzel, eds, Springer Nature, Switzerland https://doi.org/10.1007/978-3-030-11605-7.

Quigg, A., J.W. Farrington, S. Gilbert, S.A. Murawski, and V.T. John. 2021. A decade of GoMRI dispersant science: Lessons learned and recommendations for the future. Oceanography 34(1):98-111, https://doi.org/10.5670/oceanog.2021.119.

Quintana-Rizzo, E., J. Torres, S. Ross, I. Romero, K. Watson, E. Goddard, and D. Hollander. 2015. $\delta^{13} \mathrm{C}$ and $\delta^{15} \mathrm{~N}$ in deep-living fishes and shrimps after the Deepwater Horizon oil spill, Gulf of Mexico. Marine Pollution Bulletin 94:241-250, https://doi.org/10.1016/j.marpolbul.2015.02.002.

Ramseur, J.L., and C.L. Hagerty. 2013. Deepwater Horizon Oil Spill: Recent Activities and Ongoing Developments. Congressional Research Service Report R42942, 19 pp., https://digital.library.unt. edu/ark:/67531/metadc306481/m1/1/high_res_d/ R42942_2014May12.pdf.

Rullkötter, J., and J.W. Farrington. 2021. What was released? Assessing the physical properties and chemical composition of petroleum and products of burned oil. Oceanography 34(1):44-57, https://doi.org/10.5670/oceanog.2021.116.

Sea Grant Programs of the Gulf of Mexico. 2020. What to Expect in Response to an Oil Spill: A Tip Sheet for Academic Researchers. GOMSG-G-20-001, MASGP-20-037, 4 pp., http://masgc.org/oilscience/academia-responsetip-sheet.pdf.

Smokorowski, K.E., and R.G. Randall. 2017. Cautions on using the before-after-control-impact design in environmental effects monitoring programs. Facets 2:212-232, https://doi.org/10.1139/ facets-2016-0058.

Snyder, S.M., E.L. Pulster, and S.A. Murawski. 2019. Associations between chronic exposure to polycyclic aromatic hydrocarbons and health indices in Gulf of Mexico tilefish (Lopholatilus chamaeleonticeps) post Deepwater Horizon. Environmental Toxicology and Chemistry 38:2,659-2,671, https://doi.org/10.1002/etc.4583.

Sutton, T.T., T. Frank, H. Judkins, and I.C. Romero. 2020. As Gulf oil extraction goes deeper, who is at risk? Community structure, distribution, and connectivity of the deep-pelagic fauna. Pp. 403-418 in Scenarios and Responses to Future Deep Oil Spills. S.A. Murawski, C.H. Ainsworth, S. Gilbert, D.J. Hollander, C.B. Paris, M. Schüter, and D.L. Wetzel, eds, Springer Nature, Switzerland, https://doi.org/10.1007/978-3-030-12963-7.

Wade, T.L., M. Morales-McDevitt, G. Bera, D. Shi, S. Sweet, B. Wang, G. Gold-Bouchot, A. Quigg, and A.H. Knap. 2017. A method for the production of large volumes of WAF and CEWAF for dosing mesocosms to understand marine oil snow formation. Heliyon 3:e00419, https://doi.org/10.1016/ j.heliyon.2017.e00419.

Weiman, S., S.B. Joye, J.E. Kostka, K.M. Halanych, and R.R. Colwell. 2021. GoMRI insights into microbial genomics and hydrocarbon bioremediation response in marine ecosystems. Oceanography 34(1):124-135, https://doi.org/ 10.5670/oceanog.2021.121.

Weinnig, A.M., C.E. Gómez, A. Hallaj, and E.E. Cordes. 2020. Cold-water coral (Lophelia pertusa) response to multiple stressors: High temperature affects recovery from short-term pollution exposure. Scientific Reports 10:1-13, https://doi.org/ 10.1038/s41598-020-58556-9.
Xue, Z., R. He, K. Fennel, W.J. Cai, S. Lohrenz, and C. Hopkinson. 2013. Modeling ocean circulation and biogeochemical variability in the Gulf of Mexico. Biogeosciences 10:7,219-7,234 https://doi.org/10.5194/bg-10-7219-2013.

\section{CONFLICT OF INTEREST}

The authors declare that the research was conducted in the absence of any commercial or financial relationships that could be construed as a potential conflict of interest.

\section{AUTHOR CONTRIBUTIONS}

All authors contributed analysis, evaluation, and publication writing leading to this contribution. Funding for the project was primarily provided by the Gulf of Mexico Research Initiative.

\section{ACKNOWLEDCMENTS}

In particular, we thank the GoMRI Core Areas 2 and 3 "motivators," including Peter Brewer, Dick Dodge, John Shepherd, Margaret Leinen, Jürgen Rullkötter, Bill Hogarth, Bob Shipp, and Rita Colwell, for their help and encouragement. Comments and input by John Farrington were particularly helpful. The GoMR management team, American Institute of Biological Sciences, and Consortium for Ocean Leadership provided logistical support. A special thanks is due to Chuck Wilson, Michael Feldman, Callan Yanoff, and Jennifer Petitt. Three reviewers provided helpful comments for improving this manuscript.

\section{AUTHORS}

Kenneth M. Halanych (ken@auburn.edu) is Professor, Department of Biological Sciences, Molette Biology Laboratory for Environmental \& Climate Change Studies, Auburn University, Auburn, AL, USA David G. Westerholm (retired) was Director, Office of Response and Restoration, National Oceanic and Atmospheric Administration, Silver Spring, MD, USA.

\section{ARTICLE CITATION}

Halanych, K.M., and D.G. Westerholm. 2021. Considerations for scientists getting involved in oil spill research. Oceanography 34(1):112-123, https://doi.org/10.5670/oceanog.2021.120.

\section{COPYRIGHT \& USAGE}

This is an open access article made available under the terms of the Creative Commons Attribution 4.0 International License (https://creativecommons.org/ licenses/by/4.0/), which permits use, sharing, adaptation, distribution, and reproduction in any medium or format as long as users cite the materials appropriately, provide a link to the Creative Commons license, and indicate the changes that were made to the original content. 